\title{
MicroRNA-219a-2-3p modulates the proliferation of thyroid cancer cells via the HPSE/cyclin D1 pathway
}

\author{
CHUANJIA YANG ${ }^{1}$, SIYANG ZHANG $^{2}$, XIAOYING CHANG $^{3}$, \\ YONGLIAN HUANG ${ }^{1}$, DONGXU CUI ${ }^{1}$ and ZHEN LIU ${ }^{1}$
}

\author{
${ }^{1}$ Department of General Surgery, Shengjing Hospital of China Medical University, Shenyang, Liaoning 110004; \\ ${ }^{2}$ Science and Experiment Center, China Medical University, Shenyang, Liaoning 110122; ${ }^{3}$ Department of Pathology, \\ Shengjing Hospital of China Medical University, Shenyang, Liaoning 110004, P.R. China
}

Received September 26, 2020; Accepted March 25, 2021

DOI: $10.3892 / \mathrm{etm} .2021 .10091$

\begin{abstract}
Heparanase (HPSE) is an endo- $\beta$-D-glucuronidase overexpressed in different types of human cancer, and a predicted target of microRNA (miRNA/miR)-219a-2-3p in thyroid cancer. The present study aimed to investigate the potential role of HPSE and miR-219a-2-3p in thyroid cancer, and the molecular mechanism of miR-219a-2-3p regulating the proliferation of thyroid cancer cells via HPSE was confirmed. Immunohistochemistry analysis was performed to detect HPSE expression in thyroid cancer sections. In addition, reverse transcription-quantitative PCR analysis was performed to detect mRNA and miR-219a-2-3p expression levels in thyroid cancer samples and cell lines. miR-219-2-3p mimic or HPSE plasmid were transfected into B-CPAP and TPC-1 thyroid cancer cells. Furthermore, western blot analysis was performed to detect the protein expression levels of HPSE and cyclin D1. Cell cycle analysis was performed using propidium iodide staining and flow cytometry, and EdU incorporation was performed to detect cell proliferation. The results demonstrated that high HPSE expression was significantly associated with tumor size, extracapsular invasion and lymph node metastasis. Notably, a statistically negative correlation was observed between HPSE mRNA expression and miR-219a-2-3p expression in thyroid cancer tumors, as well as in thyroid cancer cell lines. When exogenously expressed in B-CPAP and TPC-1 cells, miR-219a-2-3p induced cell cycle arrest at the $G_{0} / G_{1}$ phase and decreased the percentage of proliferating cells. Furthermore, HPSE and cyclin D1 protein expression decreased following transfection with miR-219a-2-3p. Notably, when HPSE was ectopically expressed in miR-219a-2-3p transfected cells, cyclin D1 expression and the number of proliferative cells increased. Taken together, these results suggest that HPSE
\end{abstract}

Correspondence to: Professor Zhen Liu, Department of General Surgery, Shengjing Hospital of China Medical University, 36 Sanhao Street, Shenyang, Liaoning 110004, P.R. China

E-mail: liuzhen1973@aliyun.com

Key words: heparanase, microRNA-219a-2-3p, thyroid cancer, cell cycle, proliferation contributes to the proliferation of thyroid cancer cells. In addition, miR-219a-2-3p was confirmed to target HPSE and inhibit cell proliferation, which was associated with cyclin D1 suppression-mediated cell cycle arrest.

\section{Introduction}

Heparanase (HPSE) is an endo- $\beta$-glucuronidase that promotes the invasion and metastasis of tumor cells (1). HPSE has been confirmed to facilitate the proliferation and metastasis of ovarian cancer (2), and downregulation of HPSE inhibits the adhesive and aggressive properties of hepatocellular carcinoma cells (3). HPSE is also an important regulator in the tumor microenvironment, such as tumor angiogenesis. Notably, HPSE mRNA expression is negatively associated with the prognostic factors of patients with pancreatic ductal adenocarcinoma. Furthermore, HPSE affects vascular endothelial growth factor $\mathrm{C}$ expression and promotes the invasion of BxPC-3 cells (4). HPSE is a key protein influencing tumor angiogenesis and cell proliferation and invasion in cervical cancer, potentially via the nuclear factor-Kappa B (NF- $\mathrm{B})$ signaling pathway (5). In a thyroid carcinoma study, HPSE2 expression was upregulated and tumors exhibited a typical combination of positive labeling for neoplastic cells and negative immunostaining in colloid (6).

The molecular mechanisms underlying the progression of different tumors by HPSE regulation can be multifaceted. It has been reported that HCCLM3 cells with high HPSE expression exhibit higher transendothelial migration (TEM) rates (7). Furthermore, downregulation of HPSE or inhibition of its activity suppresses TEM in HCC cells. The role of HPSE in modulating autophagy was established in normal and malignant cells, thereby conferring growth advantages, as well as resistance to chemotherapy (7). MicroRNA (miRNA/miR)-558 facilitates the progression of gastric cancer by directly targeting the HPSE promoter to attenuate SMAD4-mediated repression of HPSE expression (8).

Currently, the biological function and molecular mechanism of miR-219a-2-3p in cancer remain unclear. miR-219a-2-3p expression is upregulated in neural stem cell-derived exosomes, which forms in the presence of insulin growth factor-1 (9). Consequently, the NF- $\kappa \mathrm{B}$ pathway is inhibited and neuroinflammation is attenuated. In addition, miR-219a-2-3p expression is 
downregulated in pituitary adenomas, which suppresses proliferation and promotes apoptosis of pituitary adenoma cells (10). Similarly, miR-219a-2-3p is expressed at low levels and negatively associated with the proliferation of gastric cancer cells (11). Our preliminary bioinformatics analysis suggested that HPSE may be a probable target of miR-219a-2-3p (data not shown). However, the function of miR-219a-2-3p in thyroid cancer and its regulation of HPSE are yet to be investigated.

The present study aimed to investigate the potential role of HPSE and miR-219a-2-3p in thyroid cancer, and to elucidate the molecular mechanism by which miR-219a-2-3p regulates the proliferation of thyroid cancer cells via HPSE.

\section{Materials and methods}

Thyroid cancer samples. Thyroid cancer sections from 80 patients, 30 fresh thyroid cancer tissues and corresponding paracancerous tissues were collected between January 2018 and June 2018 in Shengjing Hospital of China Medical University (Shenyang, China). The inclusion criteria were as follows: i) Patients diagnosed with thyroid cancer for the first time; ii) no treatment accepted prior to surgery; and iii) a pathological diagnosis of thyroid cancer. The exclusion criteria included: i) Cases with incomplete clinicopathological data; ii) patients who received radiotherapy or chemotherapy before surgery; and iii) patients with a previous history of thyroid surgery. The 80 cases comprised 21 male and 59 female patients with a mean age of 51 years (range, 32-78 years). All samples were surgical resections and the paracancerous tissues were collected at least $2 \mathrm{~cm}$ away from the tumor edge. The sections were placed at room temperature and the fresh tissues were preserved in liquid nitrogen until subsequent experimentation.

Formalin-fixed paraffin-embedded (FFPE) thyroid cancer sections were stained at room temperature with hematoxylin for $10 \mathrm{~min}$ and eosin (H\&E) for $2 \mathrm{~min}$, and diagnosed according to the 4th edition of World Health Organization classification of endocrine tumors guidelines (12) and the 8th edition of the American Joint Committee on Cancer (AJCC)/TNM staging system of thyroid cancer (13), by two senior pathologists. The clinicopathological characteristics of the 80 patients, including sex, age, differentiation, tumor size, extracapsular invasion and lymph node metastasis are presented in Table I. The present study was approved by the Medical Research Ethics Committee of China Medical University (ethics approval no. 2014PS47K) and written informed consent was provided by all patients prior to the study start.

Immunohistochemistry. A total of 80 thyroid cancer samples were fixed with $10 \%$ formaldehyde at room temperature for $10 \mathrm{~min}$, embedded in paraffin, and 4- $\mu \mathrm{m}$ thick sections were prepared. These sections were deparaffinized and conventionally rehydrated. Following antigen recovery, the sections were incubated with $3 \% \mathrm{H}_{2} \mathrm{O}_{2}$ to inhibit endogenous peroxidase activity, followed by $5 \%$ non-immune goat serum at $37^{\circ} \mathrm{C}$ for $30 \mathrm{~min}$ to block the unspecific antibody binding. Sections were incubated with rabbit polyclonal antibody specific for HPSE (1:200; 24529-1-AP; ProteinTech Group, Inc.) at $4^{\circ} \mathrm{C}$ overnight. After $24 \mathrm{~h}$, tissue sections were incubated with goat anti-rabbit IgG (1:1,000; KIT-0105R; Maxim Biotechnologies, Inc.) and streptavidin-peroxidase (SP) complex at $37^{\circ} \mathrm{C}$ for $30 \mathrm{~min}$
(KIT-9710; Maxim Biotechnologies, Inc.), and subsequently stained with 3,3'-diaminobenzidine at room temperature for a few seconds. The non-immune goat IgG (1:200 dilution; KIT-0105R; Maxim Biotechnologies, Inc.) was used as the negative control instead of primary antibody. A total of two senior pathologists separately evaluated the immunostained sections under an optical microscope. Brown particles in the cytoplasm were considered positive HPSE expression. The intensity of HPSE staining was determined as follows: 0, negative; 1 , weak and 2 , intense. The percentage of positive cells $(\leq 50 \%, 1 ;>50 \%, 2)$ were assessed in at least five randomly selected fields (magnification, x400) and both values were multiplied to obtain a final score for each section as $0,1,2$ or 4. The sections were classified into low expression (score $\leq 2$, including score 0 ) or high expression (score $>2$ ).

Cell culture and transfection. Human normal Nthy-ori 3-1 thyrocytes and the thyroid cancer cell lines, B-CPAP, TPC-1, KTC-1 and K1, were purchased from the Cell Bank of Type Culture Collection of The Chinese Academy of Sciences and maintained in RPMI-1640 medium (Gibco; Thermo Fisher Scientific, Inc.) supplemented with $10 \%$ fetal bovine serum (BioInd, Inc.), at $37^{\circ} \mathrm{C}$ with $5 \% \mathrm{CO}_{2}$. The B-CPAP and $\mathrm{K} 1$ cell lines were authenticated via short tandem repeat profiling, provided by the Cell Line Authentication Service from Shanghai Blowing Biotechnology Co., Ltd (http://www.biowing.com.cn/). miR-219a-2-3p mimic and miR-negative control (NC) were purchased from Shanghai GeneChem Co., Ltd. The sequence of miR-219a-2-3p was 5'-AGA AUUGUGGCUGGACAUCUGU-3' and the NC sequence was 5'-ACGUCGUCACCGGCUAGCAGCAC-3'. Cells were transfected with $50 \mathrm{nM}$ miR-219a-2-3p or miR-NC using Lipofectamine ${ }^{\circledR} 3000$ (Invitrogen; Thermo Fisher Scientific, Inc.) at $37^{\circ} \mathrm{C}$ for $24 \mathrm{~h}$. The plasmids for HPSE and the empty vector were purchased from OBiO Technology (https://www.obiosh.com). The plasmids were transfected into B-CPAP or TPC-1 cells using Lipofectamine ${ }^{\circledR} 3000$ (L3000008; Invitrogen; Thermo Fisher Scientific, Inc.) $24 \mathrm{~h}$ after miR-219a-2-3p or miR-NC transfection at $37^{\circ} \mathrm{C}$ for another $24 \mathrm{~h} .48 \mathrm{~h}$ later, cells were applied for subsequent experimentation. All experiments were performed in triplicate.

Reverse transcription-quantitative $(R T-q) P C R$. Total RNA from fresh thyroid tumors and corresponding paracancerous tissues or cultured thyroid cancer cells was extracted using the RNApure kit (Aidlab; (http://www.aidlab.cn), and RNA concentration and purity was determined by UV spectroscopy. The RNA was then reverse transcribed into cDNA at $25^{\circ} \mathrm{C}$ for 5 min and $42^{\circ} \mathrm{C}$ for $1 \mathrm{~h}$ using the GoScript Reverse Transcription System (Promega Corporation). qPCR was subsequently performed using the GoTaq qPCR Master Mix (Promega Corporation) in the Roche LightCycler 480 Real-Time PCR instrument. The following primer sequences were used for qPCR: HPSE forward, 5'-AGTGGGTGTGGGTGATTTCC-3' and reverse, 5'-GGCTCCTGGGTGAAGAAGTC-3'; GAPDH forward, 5'-CAGGAGGCATTGCTGATGAT-3 and reverse, 5'-GA AGGC TGGGGC TCA TTT-3'; miR-219a-2-3p forward, 5'-GTCCAGAAT TGTGGCTGGAC-3' and reverse, 5'-GCAGGGTCCGAGGTATTC-3'; and U6 forward, 5'-CTCGCTTCGGCAGCACA-3' and reverse, 
5'-AACGCTTCACGAATTTGCGT-3'. The length of PCR products were 193 base pairs (bp), 152, 22 and $94 \mathrm{bp}$, respectively. The following thermocycling conditions were used for qPCR: 40 cycles with an initial denaturation at $95^{\circ} \mathrm{C}$ for $2 \mathrm{~min}$, amplification at $95^{\circ} \mathrm{C}$ for $15 \mathrm{sec}$ and annealing at $60^{\circ} \mathrm{C}$ for $1 \mathrm{~min}$. Relative expression levels were calculated using the $2^{-\Delta \Delta \mathrm{Cq}}$ (14) method and normalized to the internal reference gene GAPDH (for HPSE) or U6 (for miR-219a-2-3p).

Western blotting. The cultured cells were lysed using RIPA containing protease inhibitor (G6521; Promega Corporation) and centrifuged at $22,000 \mathrm{x} \mathrm{g}$ at $4^{\circ} \mathrm{C}$ for $30 \mathrm{~min}$ for supernatant extraction. The protein content was determined using a BCA assay kit (Beyotime Institute of Biotechnology). Total protein $(50 \mu \mathrm{g})$ was separated by $12 \%$ SDS-PAGE and then transferred to a PVDF membrane. The membranes were incubated with $5 \%$ non-fat milk powder at room temperature for $2 \mathrm{~h}$ to block nonspecific antibody binding. The membranes were then incubated with the following primary antibodies: Rabbit anti-HPSE (1:1,000; cat. no. 24529-1-AP; ProteinTech Group, Inc.), mouse anti-cyclin D1 (1:500; cat. no. TA804673; ZSGB-BIO) and anti-GAPDH (1:500; cat. no. TA-08; ZSGB-BIO) overnight at $4^{\circ} \mathrm{C}$. Following the primary incubation, membranes were incubated with goat anti-rabbit (1:4,000; cat. no. ZB-2301) or anti-mouse $\operatorname{IgG}(1: 2,000$; cat. no. ZB-2305) secondary antibodies at room temperature for $2 \mathrm{~h}$, which were both purchased from ZSGB-BIO. Protein bands were developed using electrochemiluminescence (WBKLS0100; MilliporeSigma) and analyzed using ImageJ software (version 1.52v; National Institutes of Health). The ratio of $\mathrm{IOD}_{\text {target protein }}$ and $\mathrm{IOD}_{\mathrm{GAPDH}}$ of the same specimen was calculated as the relative expression level of target protein.

Dual-luciferase reporter assay. The TargetScan database (http://www.targetscan.org) revealed that miR-219a-2-3p shares complementary binding sequences with HPSE. TargetScan predicts HPSE as a potential target of any miRNAs by searching for the presence of conserved 8mer sites that match the seed region, and miR-219a-2-3p (position 1603-1610 of HPSE3'-UTR) was selected in the present study. Partial HPSE sequences containing predictive miR-219a-2-3p binding sites or mutated sites [HPSE-wild-type (WT) and HPSE-mutant (MUT)] were customized by Shanghai GenePharma, Co., Ltd. B-CPAP or TPC-1 cells were transfected with miR-219a-2-3p mimic or miR-NC, with WT or MUT luciferase reporter, using Lipofectamine ${ }^{\circledR} 3000$ (Invitrogen; Thermo Fisher Scientific, Inc.). The sequence of miR-219a-2-3p was 5'-AGAAUUGUGGCUGGACAUCUGU-3' and the NC sequence was 5'-ACGUCGUCACCGGCUAGCAGCAC-3'. Following incubation at $37^{\circ} \mathrm{C}$ for $48 \mathrm{~h}$, cells were harvested, and luciferase activities were detected using the Dual-Lumi ${ }^{\mathrm{TM}}$ Luciferase Reporter Gene Assay kit (RG089S; Beyotime Institute of Biotechnology). Renilla luciferase activity was used as an internal control.

EdU incorporation assay. The kFlour555 Click-iT EdU imaging kit (Nanjing KeyGen Biotech, Co., Ltd.) was used to assess cell proliferation. Staining was performed as follows: Cells were cultured with $20 \mu \mathrm{mol} / 1$ of diluted EdU at $37^{\circ} \mathrm{C}$ for $2 \mathrm{~h}$. Cells were fixed with $4 \%$ paraformaldehyde at room temperature for $10 \mathrm{~min}$, and neutralized with a $2 \mathrm{mg} / \mathrm{ml}$ glycine solution. Cells were washed with PBS, permeated with
$0.5 \%$ Triton X-100 and subsequently incubated with prepared Click-iT reaction cocktail at room temperature for $30 \mathrm{~min}$ in the dark. DAPI was used to counterstain the nuclei at room temperature for $30 \mathrm{~min}$, and stained cells were counted in five randomly selected fields using an inverted fluorescent microscopy (magnification, x200). Cell proliferative rates were represented as red/blue.

Statistical analysis. Statistical analysis was performed using SPSS 16.0 software (SPSS, Inc.). Data are representative of three independent experiments and presented as the mean \pm standard deviation. The $\chi^{2}$ test was used to assess the association between HPSE expression and the clinicopathological characteristics of patients with thyroid cancer. Paired-samples t-test was used to compare differences between cancer tissues and paracancerous tissues. Spearman's rank correlation coefficient was used to assess the correlation between HPSE mRNA and miR-219a-2-3p expression levels in thyroid cancer tissues and cell lines. Unpaired t-test was used to compare differences between two groups, while one-way ANOVA and LSD (3 groups) or Tukey's ( $>3$ groups) post hoc tests were used to compare differences between multiple groups. $\mathrm{P}<0.05$ was considered to indicate a statistically significant difference.

\section{Results}

HPSE expression in thyroid cancer specimens via immunohistochemistry analysis. The thyroid follicular epithelial cells exhibited weak HPSE expression in the cytoplasm. HPSE immunostaining was also observed in the cytoplasm of thyroid cancer cells. Among the specimens, 60 (75.0\%) were considered high expression (scores $>2$ ), while 20 were considered low expression (scores $\leq 2$ ). Given that HPSE expression was associated with tumor growth and metastasis, the association between HPSE expression and the clinicopathological characteristics of patients with thyroid cancer was assessed. As presented in Table I, HPSE expression was significantly associated with tumor size $(\mathrm{P}=0.018)$, extracapsular invasion $(\mathrm{P}=0.006)$ and lymph node metastasis $(\mathrm{P}=0.014)$. However, no significant differences were observed between HPSE expression and sex, age or differentiation ( $\mathrm{P}>0.05)$. HPSE expression in normal thyroid follicular epithelium (Fig. 1A and B), paracancerous tissues (Fig. 1C) and thyroid cancer tissues (Fig. 1D-I) is presented in Fig. 1. The corresponding H\&E images of Fig. 1 are presented in Fig. S1.

miR-219a-2-3p expression is negatively correlated with HPSE $m R N A$ expression in thyroid cancer tissues and cell lines. HPSE was predicted as a target gene of miR-219a-2-3p, thus, HPSE mRNA and miR-219a-2-3p expression levels were detected in 30 fresh thyroid cancer tissues and four thyroid cancer cell lines, and the paracancerous thyroid tissues and normal thyrocytes were used as the controls, respectively. The results demonstrated that HPSE mRNA expression was significantly higher $(\mathrm{P}<0.01$; Fig. 2A), while miR-219a-2-3p expression was significantly lower $(\mathrm{P}<0.01$; Fig. $2 \mathrm{~B})$ in thyroid cancer tissues compared with paracancerous tissues. Furthermore, a negative correlation was observed between HPSE mRNA and miR-219a-2-3p expression in thyroid cancer tissues (r, -0.407 ; $\mathrm{P}=0.026$; Fig. 2C). Similar results were presented in thyroid cancer cell lines, whereby 
Table I. Association between heparanase expression and clinicopathological characteristics of patients with thyroid cancer via immunohistochemical staining $(n=80)$.

\begin{tabular}{|c|c|c|c|c|}
\hline Characteristic & Number of patients & Low expression $(\mathrm{n}=20)$ & High expression $(\mathrm{n}=60)$ & P-value \\
\hline \multicolumn{5}{|l|}{ Sex } \\
\hline Male & 21 & 7 & 14 & \multirow[t]{2}{*}{0.304} \\
\hline Female & 59 & 13 & 46 & \\
\hline \multicolumn{5}{|l|}{ Age, years } \\
\hline$<55$ & 30 & 10 & 20 & \multirow[t]{2}{*}{0.182} \\
\hline$\geq 55$ & 50 & 10 & 40 & \\
\hline \multicolumn{5}{|l|}{ Differentiation } \\
\hline Well & 62 & 14 & 48 & \multirow[t]{2}{*}{0.367} \\
\hline Poor & 18 & 6 & 12 & \\
\hline \multicolumn{5}{|l|}{ Tumor size, cm } \\
\hline$\leq 2$ & 37 & 14 & 23 & \multirow[t]{3}{*}{$0.018^{\mathrm{a}}$} \\
\hline$>2-\leq 4$ & 35 & 5 & 30 & \\
\hline$>4$ & 8 & 1 & 7 & \\
\hline \multicolumn{5}{|c|}{ Extracapsular invasion } \\
\hline Yes & 33 & 3 & 30 & \multirow[t]{2}{*}{$0.006^{\mathrm{b}}$} \\
\hline No & 47 & 17 & 30 & \\
\hline \multicolumn{5}{|c|}{ Lymph node metastasis } \\
\hline Yes & 43 & 6 & 37 & \multirow[t]{2}{*}{$0.014^{\mathrm{a}}$} \\
\hline No & 37 & 14 & 23 & \\
\hline
\end{tabular}

${ }^{\mathrm{a}} \mathrm{P}<0.05 ;{ }^{\mathrm{b}} \mathrm{P}<0.01$.

HPSE expression was significantly higher (Fig. 2D, $\mathrm{P}<0.01$ ), while miR-219a-2-3p expression was significantly lower (Fig. 2E, $\mathrm{P}<0.01$ ) in thyroid cancer cells compared with normal thyrocytes. In addition, a negative correlation was observed between HPSE mRNA and miR-219a-2-3p expression in thyroid cancer cells (r, $-0.720 ; \mathrm{P}=0.008$; Fig. $2 \mathrm{~F}$ ). B-CPAP and TPC-1 cells (lowest miR-219a-2-3p expression) were selected for subsequent experimentation.

miR-219a-2-3p modulates HPSE expression in B-CPAP and TPC-1 cells. Previous studies have reported the involvement of miR-219a-2-3p in cancer development $(10,11)$. Thus, it was speculated that miR-219a-2-3p participates in the progression of thyroid cancer by targeting HPSE. The TargetScan database revealed that miR-219a-2-3p shares complementary binding sequences with HPSE (Fig. 3A). The dual-luciferase reporter assay was performed to verify the interaction between HPSE and miR-219a-2-3p. miR-219a-2-3p expression was confirmed to be upregulated in B-CPAP and TPC-1 cells following transfection with miR-219a-2-3p mimic (Fig. 3B). The results demonstrated that miR-219a-2-3p mimic notably decreased the luciferase activity of HPSE reporter compared with miR-NC (Fig. 3C). However, no significant differences were observed in the luciferase activity of HPSE-MUT reporter (Fig. 3C).

HPSE mediates miR-219a-2-3p-induced cyclin D1 inhibition, cell cycle arrest at $G_{0} / G_{1}$ and cell proliferation suppression. B-CPAP and TPC-1 cells were transiently transfected with miR-NC and miR-219a-2-3p mimic, and western blot analysis was performed to detect the protein expression levels of HPSE and cyclin D1. The results demonstrated that HPSE expression increased in both B-CPAP and TPC-1 cells following transfection with HPSE plasmids (Fig. 4A). Conversely, HPSE and cyclin D1 expression levels significantly decreased following transfection with miR-219a-2-3p mimic compared with the miR-NC groups, respectively. Notably, when HPSE expression exogenously increased in miR-219a-2-3p mimic-transfected cells, cyclin D1 expression increased accordingly (Fig. 4B). Taken together, these results suggest that miR-219a-2-3p regulates cyclin D1 by targeting HPSE.

Cell cycle analysis demonstrated a higher number of B-CPAP and TPC- 1 cells in the $G_{0} / G_{1}$ phase and less cells in the $S$ phase following transfection with miR-219a-2-3p mimic compared with the miR-NC groups, respectively (Fig. 4C). However, when ectopic HPSE expression was induced in miR-219a-2-3p mimic-transfected cells, the percentage of cells decreased in the $G_{0} / G_{1}$ phase and increased in the $S$ phase compared with the empty vector-transfected cells (Fig. 4D). Collectively, these results suggest that miR-219a-2-3p induces cell cycle arrest at the $G_{0} / G_{1}$ phase, which was also associated with HPSE.

The proliferative ability of B-CPAP and TPC- 1 cells was assessed via the EdU incorporation assay. The results demonstrated that the percentage of EdU-positive B-CPAP cells was $32.4 \pm 1.4$ and $28.3 \pm 2.7 \%$ in the miR-NC and miR-219a-2-3p mimic groups, respectively $(\mathrm{P}=0.016$; Fig. $4 \mathrm{C})$. In the empty vector or HPSE transfected cells overexpressed with miR-219a-2-3p, the percentage of EdU-positive B-CPAP cells was $23.3 \pm 2.0$ and $37.2 \pm 2.3 \%$, respectively ( $<<0.01$; Fig. 4D). 


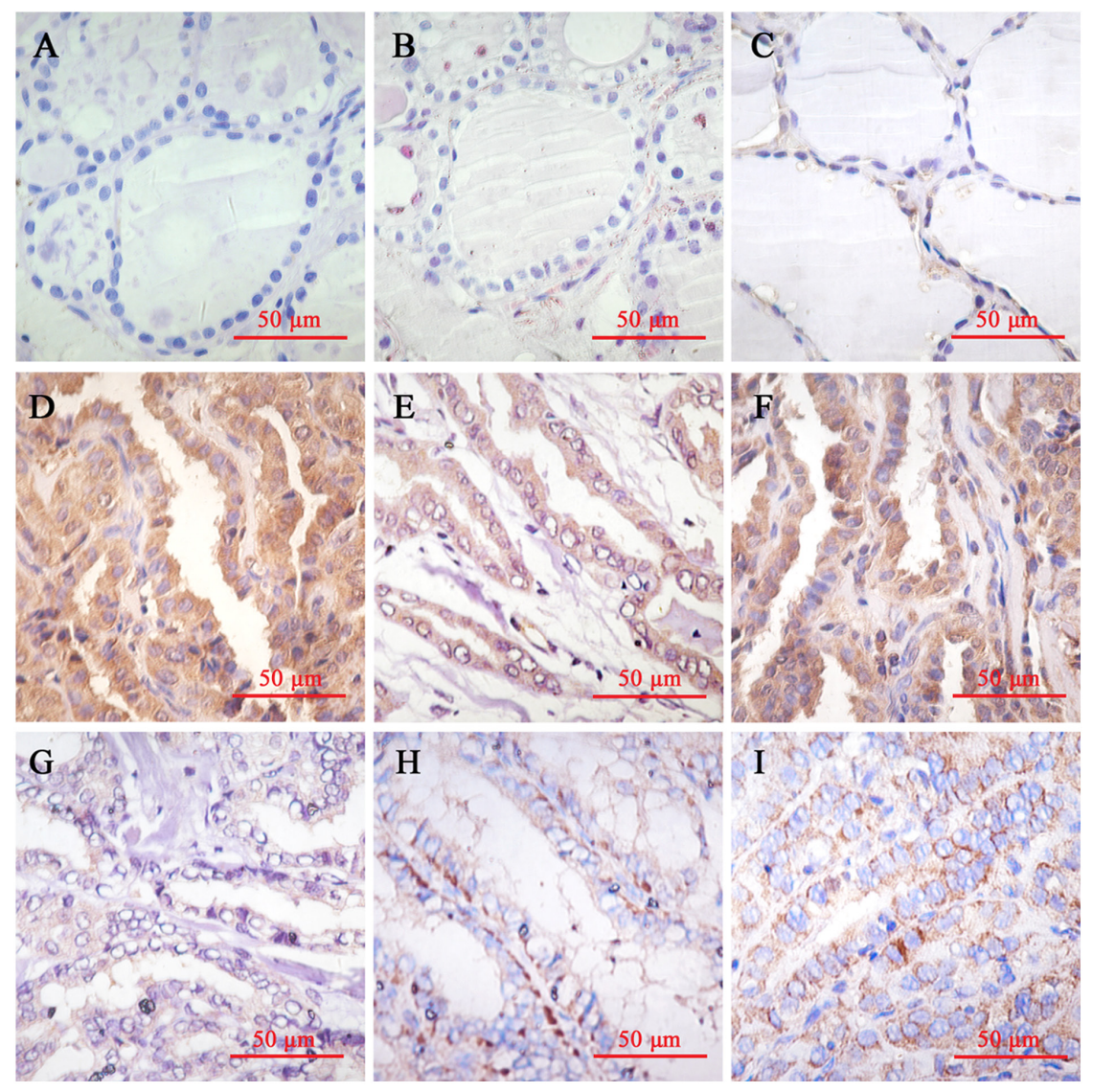

Figure 1. HPSE expression in thyroid cancer samples via immunohistochemical staining. Nuclei were counterstained with hematoxylin. HPSE expression was negative or weak in (A) thyroid follicular epithelial cells, (B) normal thyroid tissues and (C) paracancerous tissues. Thyroid cancer tissues exhibited differential HPSE expression, including positive staining in (D) tumors $>4 \mathrm{~cm}$, (E) neoplastic samples with extracapsular invasion, (F) thyroid cancer tissues with lymph node metastasis, $(\mathrm{G})$ tumors $\leq 2 \mathrm{~cm},(\mathrm{H})$ neoplastic samples without extracapsular invasion, and (I) thyroid cancer samples without lymph node metastasis. Magnification, x400. HPSE, heparanase.

Similarly, the percentage of proliferative TPC-1 cells was $30.3 \pm 1.2$ and $28.0 \pm 1.6 \%$ in the miR-NC and miR-219a-2-3p mimic groups, respectively $(\mathrm{P}=0.028$; Fig. $4 \mathrm{C})$. In the empty vector or HPSE transfected cells overexpressed with miR-219a-2-3p, the percentage of proliferative TPC-1 cells was $22.5 \pm 2.4$ and $33.5 \pm 2.5 \%$, respectively ( $\mathrm{P}<0.01$; Fig. 4D). Taken together, these results suggest that miR-219a-2-3p inhibits the proliferative ability of thyroid cancer cells via HPSE.

\section{Discussion}

HPSE expression is upregulated in different types of human cancer, which is associated with tumor metastasis and invasion $(15,16)$. It has been reported that high HPSE expression is associated with poor prognosis and contributes to the lymphovascular invasion of breast cancer (17). In a systematic study, either HPSE expression in cancer and/or serum HPSE concentration were reported to act as potential biomarkers for the evaluation of surgery effects and prognosis prediction in patients with ovarian cancer (18). In the present study, HPSE expression was investigated in 80 FFPE thyroid cancer samples via immunohistochemical staining. The results demonstrated that HPSE expression was upregulated in thyroid cancer samples, and normal thyroid follicular epithelium exhibited negative or weak HPSE staining, supporting its potential role in thyroid tumorigenesis. Furthermore, the clinical significance of HPSE in thyroid cancer was assessed. It was hypothesized that thyroid cancer with high HPSE expression was more likely to exhibit tumor extension and lymph node metastasis. Given that HPSE is critical in tumorigenesis and progression of thyroid cancer (6), repressing HPSE expression may be a promising option for inhibiting thyroid cancer. In other cancers, downregulation of HPSE represses glioma cell proliferation, and exogenous HPSE expression stimulates growth and activates ERK and AKT signaling (19). In addition, miR-429 decreases the invasive ability of gastric cancer cells by downregulating HPSE expression (20).

miRNAs are small non-coding RNA molecules that function in post-transcriptional silencing of gene expression via base pairing with complementary sequences within the target mRNAs. Bioinformatic analysis via the TargetScan database revealed that HPSE is a regulatory target of miR-219a-2-3p. Previous studies have reported that miR-219a-2-3p is a tumor suppressor in pituitary adenoma (10), glioma (21) and gastric cancer (11). Mouse double minute 2 homolog (MDM2) is a putative target of miR-219a-2-3p, based on bioinformatics analysis in pituitary adenomas. miR-219a-2-3p decreases MDM2 expression by binding to its 3'-UTR and promoting p53 expression, which in turn suppresses cell proliferation (10). In glioma cell lines with WT isocitrate dehydrogenase (IDH)1/2, overexpression of miR-219a-2-3p decreases cell proliferation and colony formation (21). The predicted targets of miR-219a-2-3p 

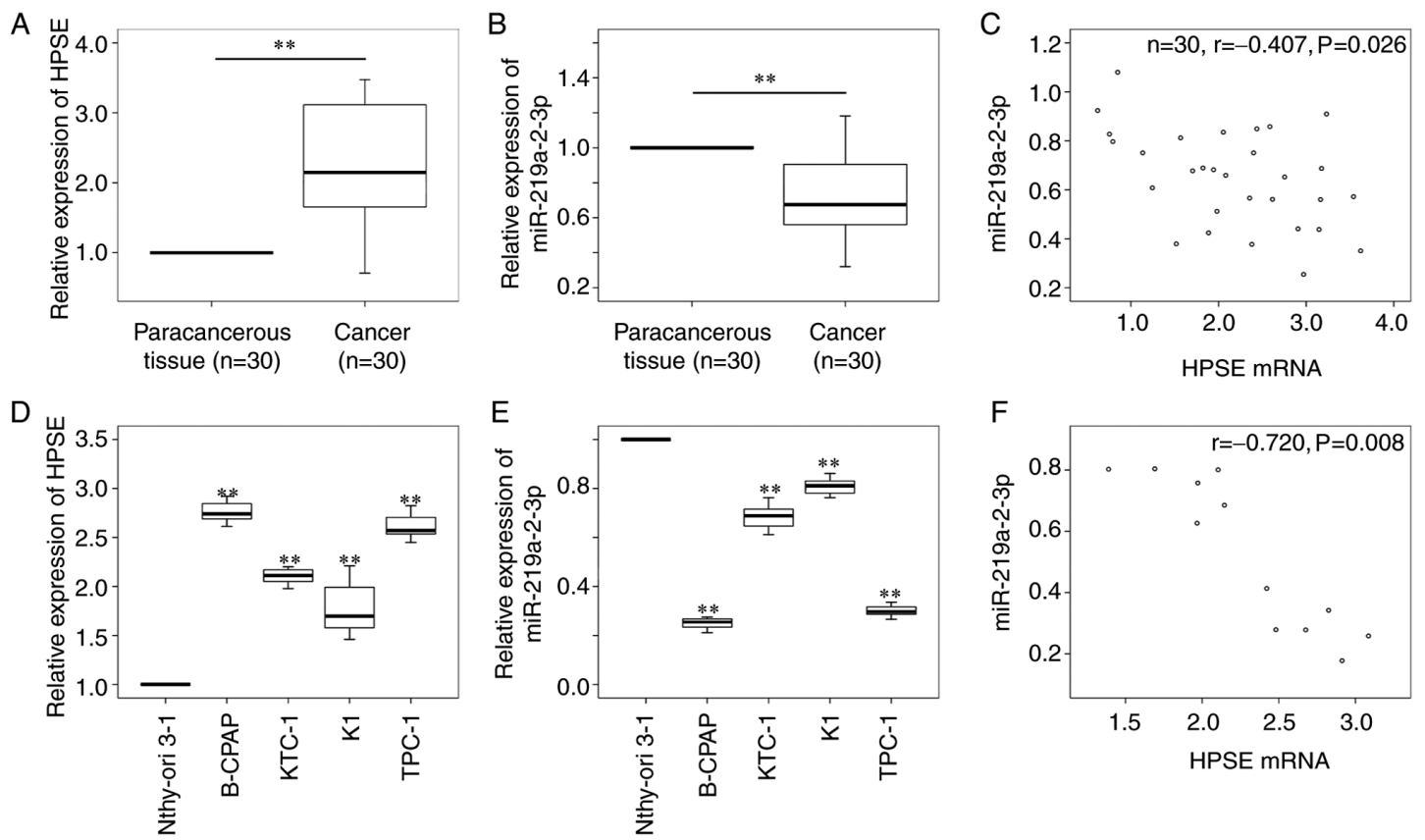

Figure 2. HPSE mRNA and miR-219a-2-3p expression in 30 fresh thyroid cancer samples and corresponding paracancerous tissues, as well as in normal thyrocytes and 4 thyroid cancer cell lines. Statistical relationship between HPSE mRNA and miR-219a-2-3p was also analyzed. (A) HPSE mRNA expression was higher in thyroid cancer tissues compared with paracancerous tissues $(n=30)$. (B) miR-219a-2-3p expression was higher in paracancerous tissues compared with thyroid cancer tissues $(\mathrm{n}=30)$. (C) HPSE mRNA and miR-219a-2-3p expression were negatively correlated in thyroid cancer tissues. (D) HPSE mRNA expression was higher in the thyroid cancer cell lines compared with normal thyrocytes. (E) miR-219a-2-3p expression was lower in the thyroid cancer cell lines compared with normal thyrocytes. (F) HPSE mRNA and miR-219a-2-3p expression were negatively correlated in thyroid cancer cell lines. ${ }^{* *} \mathrm{P}<0.01$ vs. Nthy-ori 3-1. HPSE, heparanase; miR, microRNA.

\section{A HPSE-WT $\quad 5^{\prime} \ldots$ AAA AAA UGG UUA AUG ACAAUUCA....3' \\ 3'-UGU \\ HPSE-MUT $\quad 5^{\prime}$...AAA AAA UGG UUA AUG CACCGGUG...3'}
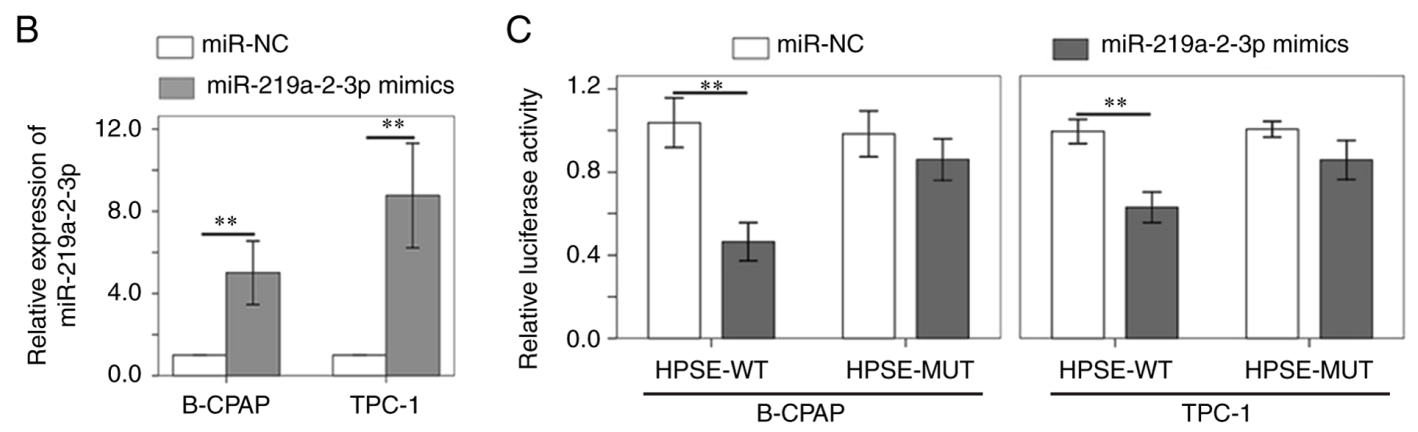

Figure 3. Potential interaction between HPSE and miR-219a-2-3p. (A) Predicted binding sites between HPSE and miR-219a-2-3p, as well as the MUT sites in HPSE-MUT reporter. (B) miR-219a-2-3p expression was upregulated in B-CPAP and TPC-1 cells following transfection with miR-219a-2-3p mimic. (C) B-CPAP and TPC-1 cells were co-transfected with miR-219a-2-3p mimic or miR-NC, with HPSE-WT or HPSE-MUT reporters, and luciferase activity was detected in each group. ${ }^{*}$ P $<0.01$. HPSE, heparanase; miR, microRNA; MUT, mutant; NC, negative control; WT, wild-type.

have been demonstrated to participate in tumor progression by activating the Ras-ERK and PI3K-AKT pathways. In addition, miR-219a-2-3p expression is downregulated in gastric cancer tissues and cells. FUS expression is negatively associated with miR-219a-2-3p. In gastric cancer cells, miR-219a-2-3p targets FUS, which facilitates cell proliferation (11).

In the present study, miR-219a-2-3p expression was downregulated in thyroid cancer tissues and cells, which was negatively correlated with HPSE mRNA expression. Based on cell cycle analysis and EdU incorporation assay,
miR-219a-2-3p mimic significantly attenuated the proliferative ability of thyroid cancer cells, which further validates its tumor suppressive role in thyroid cancer. In addition, miR-219a-2-3p decreased cyclin D1 expression and induced cell cycle arrest at the $G_{0} / G_{1}$ phase, the effects of which were reversed following exogenous HPSE expression. Taken together, these results suggest that miR-219a-2-3p negatively regulates the proliferation of thyroid cancer cells via HPSE. The regulatory mechanism of miR-219a-2-3p on HPSE was further confirmed as the 3'-UTR luciferase activity of HPSE 

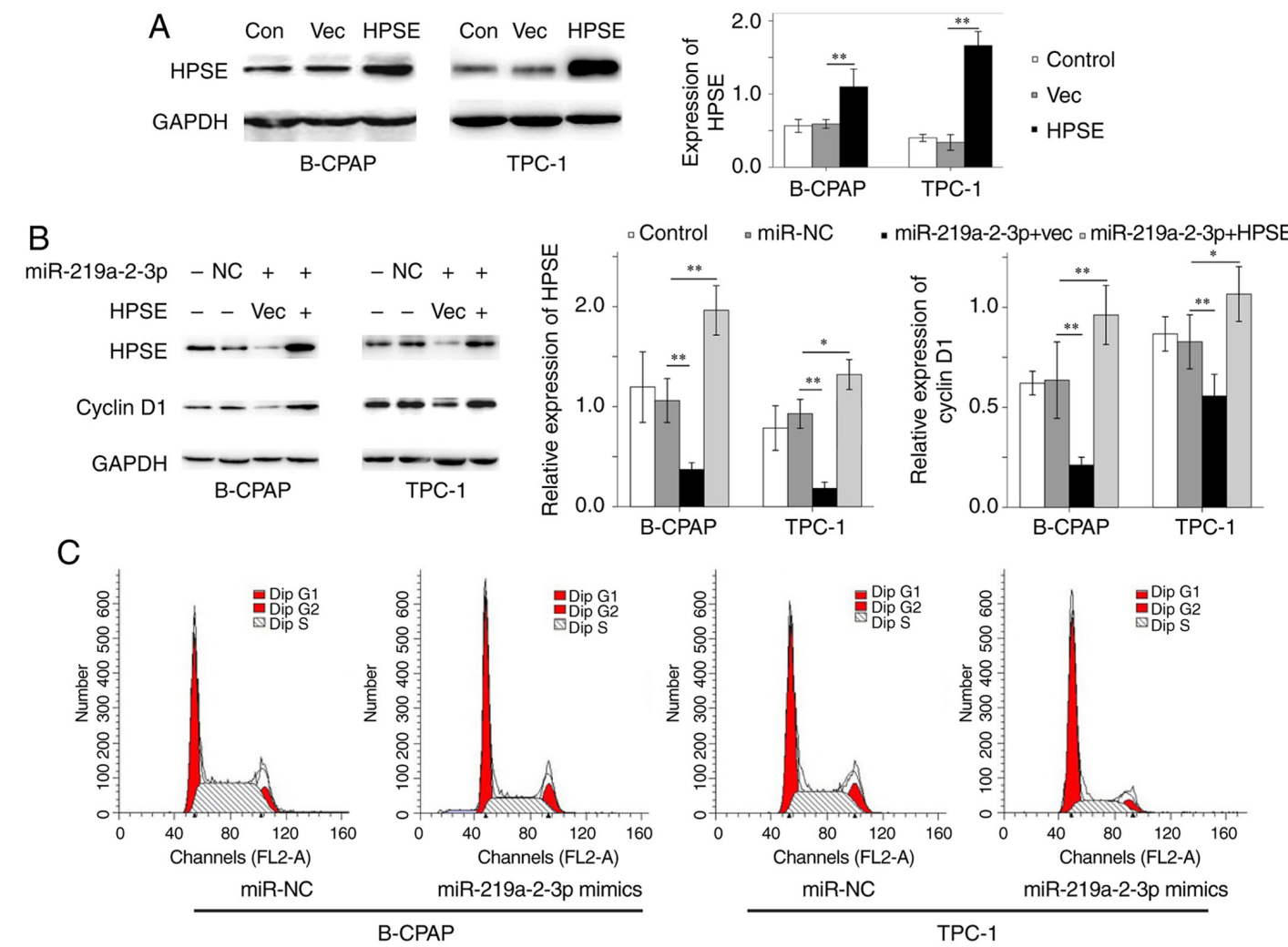

miR-NC
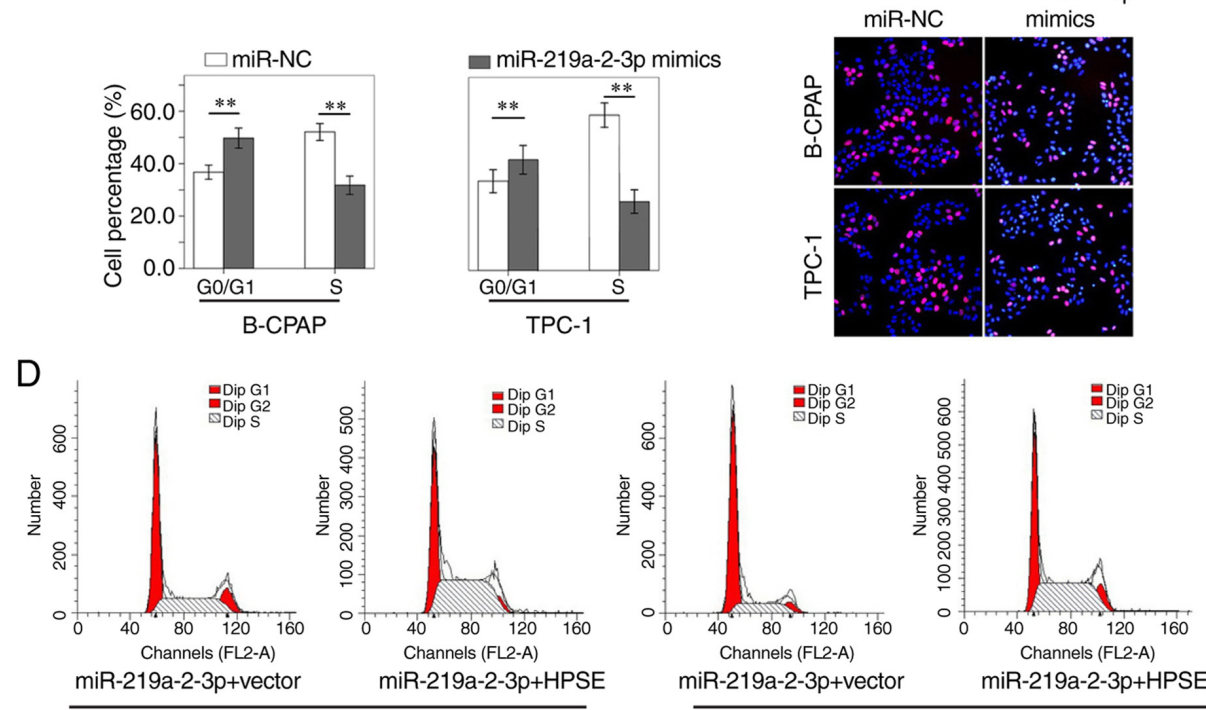

B-CPAP

miR-219a-2-3p+vector
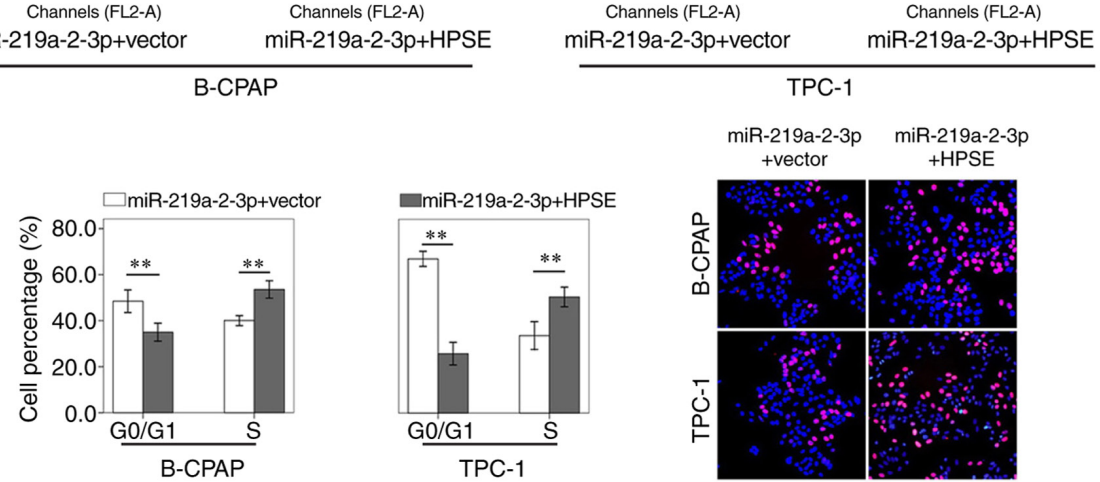

Figure 4. HPSE and cyclin D1 expression, cell cycle and cell proliferation are regulated by miR-219a-2-3p mimic via HPSE. (A) HPSE expression increased in B-CPAP and TPC-1 cells following transfection with HPSE plasmids. (B) miR-219a-2-3p mimic suppressed HPSE and cyclin D1 expression. Notably, ectopic HPSE expression reversed the inhibitory effect of miR-219a-2-3p mimic on cyclin D1 expression. (C) Cell cycle analysis indicated a higher number of B-CPAP and TPC-1 cells in the $\mathrm{G}_{0} / \mathrm{G}_{1}$ phase and less cells in the $\mathrm{S}$ phase following transfection with miR-219a-2-3p mimic. Furthermore, the number of EdU-positive B-CPAP and TPC-1 cells decreased following transfection with miR-219a-2-3p mimic. (D) HPSE was exogenously expressed in miR-219a-2-3p mimic-transfected cells, which decreased the number of cells in the $G_{0} / G_{1}$ phase and increased the number of cells in the $S$ phase compared with the empty vector group. In addition, the number of proliferative cells was increased in miR-219a-2-3p mimic-transfected cells with HPSE upregulation. Magnification, x200. Red staining, EdU; blue staining, DAPI. " $\mathrm{P}<0.05$; ${ }^{* *} \mathrm{P}<0.01$. HPSE, heparanase; miR, microRNA; NC, negative control; Con, control; Vec, empty vector. 
significantly decreased in miR-219a-2-3p mimic-transfected cells, resulting in decreased HPSE protein expression.

In conclusion, the results of the present study suggest that HPSE is essential for the development of thyroid cancer, and is the downstream target gene of miR-219a-2-3p. miR-219a-2-3p decreased the proliferative ability of thyroid cancer cells and induced cell cycle arrest at the $G_{0} / G_{1}$ phase, likely by decreasing cyclin D1 expression, which was associated with HPSE suppression. Furthermore, overexpression of miR-219a-2-3p suppressed the proliferation of thyroid cancer cells by targeting HPSE and cyclin D1, thus, the miR-219a-2-3p/HPSE/cyclin D1 axis may be a novel area in thyroid cancer research. Further studies are required to confirm the role of miR-219a-2-3p/HPSE and its downstream pathway in thyroid cancer, in addition to cyclin D1 in the regulation of thyroid cancer progression, which may provide a potential therapeutic target to neutralize the effects of HPSE on thyroid cancer. In addition, this in vitro analysis on the regulation of miR-219a-2-3p/HPSE on cell proliferation requires in vivo validation.

\section{Acknowledgements}

Not applicable.

\section{Funding}

The present study was supported by the National Natural Science Foundation of China (grant no. 81672644) and the 345 Talent Project of Shengjing Hospital (grant no. M0731).

\section{Availability of data and materials}

The datasets used and/or analyzed during the current study are available from the corresponding author on reasonable request.

\section{Authors' contributions}

CY and ZL made substantial contributions to the conception and design of the present study. SZ and $\mathrm{YH}$ performed the experiments and drafted the initial manuscript. XC and DC analyzed and interpreted the data. YH and CY confirmed the authenticity of all the raw data. All authors have read and approved the final manuscript.

\section{Ethics approval and consent to participate}

The present study was approved by the Ethics Committee of Shengjing Hospital of China Medical University (Shenyang, China; ethics approval no. 2014PS47K). Written informed consent was provided by all patients prior to the study start.

\section{Patient consent for publication}

Not applicable.

\section{Competing interests}

The authors declare that they have no competing interests.

\section{References}

1. Masola V, Zaza G, Gambaro G, Franchi M and Onisto M: Role of heparanase in tumor progression: Molecular aspects and therapeutic options. Semin Cancer Biol 62: 86-98, 2020.

2. Zheng HY, Ruan J, Zhao P, Chen SP, Pan LL and Liu JQ: Heparanase is involved in proliferation and invasion of ovarian cancer cells. Cancer Biomark 15: 525-534, 2015.

3. Chen XP, Luo JS, Tian Y, Nie CL, Cui W and Zhang WD: Downregulation of heparanase expression results in suppression of invasion, migration, and adhesion abilities of hepatocellular carcinoma cells. Biomed Res Int 2015: 241983, 2015.

4. Lv B, Zhang B, Hu XY and Zeng QD: Heparanase regulates in vitro VEGF-C expression and its clinical significance to pancreatic ductal cell adenocarcinoma. Oncol Lett 11: 1327-1334, 2016.

5. Lv QY, Wu KJ, Liu FL, Wu WR, Chen YR and Zhang W: Interleukin-17A and heparanase promote angiogenesis and cell proliferation and invasion in cervical cancer. Int $\mathrm{J}$ Oncol 53: 1809-1817, 2018.

6. Matos LL, Suarez ER, Theodoro TR, Trufelli DC, Melo CM, Garcia LF, Oliveira OC, Matos MG, Kanda JL, Nader HB, et al: The profile of heparanase expression distinguishes differentiated thyroid carcinoma from benign neoplasms. PLoS One 10: $\mathrm{e} 0141139,2015$.

7. Chen XP, Jiang W, Yue CF, Zhang WJ, Tong CG, Dai DF, Cheng B, Huang C and Lu L: Heparanase contributes to trans-endothelial migration of hepatocellular carcinoma cells. J Cancer 8: 3309-3317, 2017.

8. Zheng LD, Jiao WJ, Song HJ, Qu HX, Li D, Mei H, Chen YJ, Yang F, Li HH, Huang K and Tong QG: miRNA-558 promotes gastric cancer progression through attenuating Smad4-mediated repression of heparanase expression. Cell Death Dis 7: e2382, 2016.

9. Ma K, Xu HY, Zhang J, Zhao F, Liang HQ, Sun HT, Li P, Zhang S, Wang RJ and Chen XY: Insulin-like growth factor-1 enhances neuroprotective effects of neural stem cell exosomes after spinal cord injury via an miR-219a-2-3p/YY1 mechanism. Aging (Albany NY) 11: 12278-12294, 2019.

10. Wang YB, Zhao JN, Zhang CC, Wang PC, Huang CX and Peng H: MiR-219a-2-3p suppresses cell proliferation and promotes apoptosis by targeting MDM2/p53 in pituitary adenomas cells. Biosci Biotechnol Biochem 84: 911-918, 2020.

11. YangZ,DongXH,PuML, YangHW,Chang WL,JiFH,LiuT,WeiCQ, Zhang XF and Qiu XG: LBX2-AS1/miR-219a-2-3p/FUS/LBX2 positive feedback loop contributes to the proliferation of gastric cancer. Gastric Cancer 23: 449-463, 2020.

12. Lloyd RV, Osamura RY, Klöppel G and Rosai J (eds): WHO Classification of Tumours of Endocrine Organs. 4th edition. IARC, Lyon, 2017.

13. Amin MB, Edge S, Greene F, Byrd DR, Brookland RK, Washington MK, Gershenwald JE, Compton CC, Hess KR, Sullivan DC, et al: AJCC Cancer Staging Manual. 8th edition. Springer, New York, NY, 2017.

14. Livak KJ and Schmittgen TD: Analysis of relative gene expression data using real-time quantitative PCR and the 2(-Delta Delta C(T)) method. Methods 25: 402-408, 2001.

15. Wang C, Wei YJ, Wang G, Zhou YM, Zhang JC and Xu K: Heparanase potentiates the invasion and migration of pancreatic cancer cells via epithelial-to-mesenchymal transition through the Wnt/ $\beta$-catenin pathway. Oncol Rep 44: 711-721, 2020.

16. Chen XP, Cheng B, Dai DF, Wu YH, Feng ZW, Tong CG, Wang XM and Zhao J: Heparanase induces necroptosis of microvascular endothelial cells to promote the metastasis of hepatocellular carcinoma. Cell Death Discov 7: 33, 2021.

17. Tang DB, Piao Y, Zhao S, Mu XD, Li S, Ma WJ, Song Y, Wang JX, Zhao WH and Zhang QY: Expression and correlation of matrix metalloproteinase-9 and heparanase in patients with breast cancer. Med Oncol 31: 26, 2014

18. Zhang W, Chan H, Wei LW, Pan ZM, Zhang JQ and Li L: Overexpression of heparanase in ovarian cancer and its clinical significance. Oncol Rep 30: 2279-2287, 2013.

19. Kundu S, Xiong AQ, Spyrou A, Wicher G, Marinescu VD, Edqvist PHD, Zhang L, Essand M, Dimberg A, Smits A, et al: Heparanase promotes glioma progression and is inversely correlated with patient survival. Mol Cancer Res 14: 1243-1253, 2016.

20. Sheng N,Zhang L and Yang SF: MicroRNA-429 decreases the invasion ability of gastric cancer cell line BGC- 823 by downregulating the expression of heparanase. Exp Ther Med 15: 1927-1933, 2018

21. Fleming J, Bell EH, Tong ZY, Grozdic I, McElroy J, Beyer S, Fabian D, Cui TT, Popp I, Staszewski O, et al: CSIG-21. The role of miR-219a-2-3p as a tumor suppressor in IDH1/2-wild-type grade II/III gliomas. Neuro Oncol 20 (Suppl 6): vi47, 2018. 